\title{
Caraterização Da Movimentação Espontânea De Lactentes Da Comunidade De Paraisópolis Em São Paulo
}

\section{Characterization Of The Spontaneous Movement Of Infants In The Paraisópolis Community In São Paulo}

\author{
DOI: $10.46919 / \operatorname{archv2n3-030}$
}

Recebimento dos originais: 01/01/2021

Aceitação para publicação: 31/03/2021

\author{
Dra. Aparecida Praeiro dos Santos \\ Fisioterapeuta. Mestranda em Medicina pela Universidade Federal de São Paulo - UNIFESP \\ E-mail: cidapraeiro@uol.com.br
}

\section{Dra. Dafne Herrero}

Fisioterapeuta. Pós doutorado pela Faculdade de Saúde Pública da USP

Av. do Café, 130, cj 81. Vila Guarani. CEP: 04311-000 São Paulo/SP

E-mail: dafneherrero@drabrincadeira.com

\section{RESUMO}

Introdução: $\mathrm{O}$ desenvolvimento neuromotor infantil constitui o resultado do aprendizado e das habilidades adquiridas na execução dos atos. Escalas de avaliação do desenvolvimento motor têm sido utilizadas, mundialmente, na tentativa de identificar através da movimentação espontânea das crianças se as mesmas apresentam sinais indicativos de alterações no desenvolvimento neuromotor. Objetivo: Avaliar a movimentação espontânea de lactentes moradores da comunidade de Paraisópolis atendidos em serviço de follow-up do Programa Einstein na Comunidade de Paraisópolis (PECP), em São Paulo. Método: Trata-se de um estudo transversal, observacional e descritivo realizado em duas etapas: a) avaliação do lactente através da verificação do desempenho motor através da Escala Motora Infantil Alberta e b) aplicação de questionário feito aos cuidadores para investigação de informações perinatais, socioeconômicas, nutricionais e de ambientes de estimulação. Foram avaliados os lactentes com idade entre 4 e 6 meses, nascidos de parto único, com peso ao nascer de 2.500, a termo, idade gestacional entre 37 e 42 semanas, moradores da comunidade de Paraisópolis em serviço de follow-up do (PECP), em São Paulo, SP no primeiro semestre de 2015. Resultados e discussão: A população de lactentes em sua maioria foi composta por meninos nascidos termo, com peso de nascimento de 3.1 gramas, AIG, seus cuidadores principais são as próprias mães. Os bebês não apresentavam nenhuma doença à época da pesquisa, foram amamentados por um período superior à 120 dias e o local de permanência dos bebês quando acordados, na grande maioria, está em berço, banheira, carinhos, colo ou no sofá. As mães tem em média 23,7 anos de idade, com escolaridade cursada em torno de 8 anos de estudo, realizaram pré-natal, sem intercorrências significativas durante o pré-natal, gestação ou para os bebês. Conclusão: a maioria dos lactentes avaliados apresentou-se abaixo da média no que se refere ao desempenho motor nos permitindo considerar que a maioria deles permanece em locais que não possibilitam movimento (berço, banheira, carrinho, colo e sofá) concordando com a literatura que crianças que crescem em ambientes carentes de estímulos não apresentam facilidade para o aprendizado motor uma vez que não possuem estruturas operacionais aptas a equacionarem novos eventos e sequências.

Palavras-chave: Desenvolvimento neuromotor. Intervenção precoce. Regionalização. Follow-up. 


\section{ABSTRACT}

Introduction: The neuromotor development of children is the result of learning and skills acquired in the execution of acts. Evaluation scales of motor development have been used, worldwide, in an attempt to identify through the children's spontaneous movement if they present indicative signs of alterations in the neuromotor development. Objective: To evaluate the spontaneous movement of infants living in the Paraisópolis community, treated in the follow-up service of the Einstein Program in Paraisópolis Community (PECP), in São Paulo. Method: This is a cross-sectional, observational and descriptive study carried out in two stages: a) evaluation of the infant by checking motor performance using the Alberta Infant Motor Scale and b) application of a questionnaire made to caregivers to investigate perinatal, socioeconomic, nutritional and stimulation environment information. Infants aged between 4 and 6 months, born from single birth, with birth weight of 2,500, at term, gestational age between 37 and 42 weeks, residents of the Paraisópolis community in follow-up service of the (PECP), in São Paulo, SP in the first half of 2015 were evaluated. Results and discussion: The population of infants was mostly composed of boys born full-term, with birth weight of 3.1 grams, AIG, their main caregivers are the mothers themselves. Infants had no diseases at the time of the survey, were breastfed for a period longer than 120 days, and the place where infants stay when awake, in the vast majority, is in the crib, bath, cuddle, lap, or on the couch. The mothers are on average 23.7 years old, with 8 years of schooling, had prenatal care, with no significant complications during prenatal care, pregnancy or for the babies. Conclusion: most of the infants assessed were below average in terms of motor performance, allowing us to consider that most of them remain in places that do not allow movement (cradle, bathtub, stroller, lap, and sofa). This agrees with the literature that children who grow up in environments lacking stimuli do not have an easy time with motor learning, since they do not have operational structures capable of equating new events and sequences.

Keywords: Neuromotor development. Early intervention. Regionalization. Follow-up.

\section{INTRODUÇÃO}

“A criança só sabe viver a sua infância, conhecê-la pertence ao adulto” diz Wallon (1998, p.27). O que nos interessa como pesquisadores, a partir deste pensamento, é identificar o que vai prevalecer nesse conhecimento: o ponto de vista do adulto ou da criança?

Esse pensamento de Henry Wallon, (1998), nos chama atenção, principalmente, nos dias atuais, pois, tendo em vista o ambiente como um fator determinante do desenvolvimento, podem-se citar as oportunidades oferecidas por ele e pela rotina do lactente e da criança, às chamadas práticas maternas, como fatores essenciais na construção do processo evolutivo, gradual e individual do desenvolvimento infantil.

Connolly, (2000), acrescenta que há a necessidade não apenas de saber o que muda e quando muda no desenvolvimento da parte motora, mas como acontecem estas mudanças e a descrição do fenômeno passa a interessar para os pesquisadores interessados no desenvolvimento infantil e principalmente para os profissionais de follow-up.

$\mathrm{Na}$ abordagem atual sobre o desenvolvimento infantil, alguns autores, como: Haidar et al, 2001; Rezende et al, 2010; Amorim et al ,2010; De Vries, 1999; Santos, 2001; Capute, 1985; Mei, 1994; Gandra,1981 e Silva et al, 2006 destacam serem importantes levar em consideração questões, como:

a) Dados maternos e paternos 
b) Moradia

c) Dados da gestação

d) Dados de parto

e) Dados neonatais

f) Condições de saúde

g) Situação de estimulação

Essas questões apontadas, pelos autores, são relevantes no momento em que consideramos que a interação do individuo no meio em que vive dão os alicerces para o desenvolvimento do raciocínio da criança e esse alicerce nada mais é que uma "reconstrução endógena de dados exógenos fornecidos pela experiência", afirma Gandra (1981). O ritmo para ordenar as informações recebidas de experiências dependerá da densidade de estimulação que o ambiente oferece. Quanto mais ordenadas estão as informações, mais acelerado é o ritmo do desenvolvimento. Quanto mais estimulante for o ambiente, maior o desenvolvimento intelectual, afirma a autora.

Crianças que crescem em ambientes carentes de estímulos não apresentam facilidade para o aprendizado uma vez que não possuem estruturas operacionais aptas a equacionarem novos eventos e sequências (GANDRA, 1981).

Portanto, entender como as mudanças motoras podem ser facilitadas pelo meio, cuidadores e acesso a informação pode tornar os dois primeiros anos de vida, deste lactente, um período de ricas descobertas, vivências e aprendizado.

Pesquisas relatam que as diferenças no desenvolvimento motor de lactentes também podem surgir na presença de variações nas práticas maternas, por sua vez as práticas maternas se modificam de acordo com o costume ou cultura de cada local. (CAPUTE 1985; MEI, 1994; DE VRIES, 1999; HAIDAR ET AL, 2001; SANTOS, 2001; SILVA ET AL, 2006; REZENDE ET AL, 2010 E AMORIM ET AL, 2010)

O desenvolvimento motor constitui, portanto, o resultado do aprendizado e das habilidades adquiridas na execução dos atos. A oportunidade para a prática é dada pelos pais ou cuidadores do lactente, através de hábitos culturais, do ambiente onde está inserida ou do treino específico de alguma habilidade.

Pesquisas como as de Connolly, 2000; Hallal, Marques e Braccialli, 2008 afirmam que lactentes nortes americanos passam mais tempo na posição de prono comparados aos lactentes britânicos, que são colocados mais frequentemente na posição supina para brincar.

O tempo de permanência em uma determinada postura influência positivamente a aquisição dos ganhos motores naquela postura, enquanto as habilidades em outras posturas podem ocorrer ligeiramente mais tarde. (CONNOLLY, 2000; HALLAL, MARQUES E BRACCIALLI, 2008) 
Lactentes britânicos demonstram precocidade nas habilidades de sentar sozinho e ficar em pé sem apoio e atraso nas habilidades de levantar a cabeça e engatinhar, comparados aos americanos, afirmam as pesquisas de Connolly, 2000; Hallal, Marques e Braccialli, 2008

O costume materno em estimular a postura sentada (britânicos) favorece o ganho motor nesta postura, e a falta de costume em posicionar o lactente em prono (americanos) pode atrasar as habilidades de apoio nas mãos e engatinhar.

Diversas escalas de avaliação do desenvolvimento motor têm sido utilizadas, mundialmente, na tentativa de identificar se as crianças apresentam sinais indicativos de alterações no desenvolvimento motor. Entre elas a Alberta Infant Motor Scale (AIMS): Escala Motora Infantil Alberta.

A AIMS, de avaliação observacional, construída por dois fisioterapeutas canadenses: Piper e Darrah, em 1994, avalia o desenvolvimento motor grosso de lactentes desde o nascimento até os 18 meses de vida. Dentre seus objetivos estão:

(1) identificar restrição do desenvolvimento neuromotor dos lactentes;

(2) informar aos pais sobre as atividades motoras que o lactente realiza ou que não realiza e aquelas que estão se desenvolvendo;

(3) analisar o desenvolvimento motor em determinado tempo ou pré e pós internação;

(4) mensurar mudanças no desenvolvimento motor que não podem ser identificadas por métodos mais tradicionais;

(5) agir como instrumento de pesquisa para identificar eficácia em programas de estimulação para lactentes com distúrbios motores.

O foco é a avaliação do desenvolvimento sequencial do controle postural referente às posições supina, prona, sentada e ortostática.

A administração do teste envolve uma avaliação observacional com o mínimo de manuseio em um tempo de 20 minutos, aproximadamente.

Segundo Herrero, et al, (2011), A AIMS tem como objetivo avaliar o desenvolvimento motor amplo ao longo do tempo dos recém-nascidos a termo e dos pré-termo de 0 a 18 meses de idade, pois a escala é uma medida observacional da performance motora infantil (movimentação espontânea) que aborda conceitos do desenvolvimento motor, como maturação do sistema nervoso central, perspectiva da dinâmica motora e avaliação da sequência do desenvolvimento motor.

A AIMS traduzida para o português contém 58 itens agrupados nas quatro posições: prono (21 itens), supino (9 itens), sentada (12 itens) e em pé (16 itens), que descrevem o desenvolvimento de movimentação espontânea e das habilidades motoras da criança. Conforme a metodologia da AIMS, não é necessário seguir uma sequência padronizada completando todos os itens de uma posição, antes de partir para a observação de outra. Conforme os movimentos apresentados no repertório da criança, para cada item 
observado ou não observado, registra-se as pontuações 1 (um) e 0 (zero), respectivamente. Ao final, somamse os valores obtidos, resultando em quatro subtotais.

Segundo Herrero et al, (2011), a AIMS:

[...] é um teste de critério de referência, com classificação percentual normatizada para permitir a determinação de onde o indivíduo se encontra em uma medida de habilidade ou traço comparado com aqueles do grupo referente.

(HERRERO, et al, 2011)

A AIMS é adequada para prover mensuração quantitativa e qualitativa do desenvolvimento motor, de crianças que desenvolveram ou não, comportamento de risco, quer no pré, no intra ou nos pós-parto.

A versão em português, traduzida em 2007, da Alberta Infant Motor Scale demonstrou ser válida e fidedigna na avaliação das aquisições motoras de crianças brasileiras, o que a torna um instrumento útil a várias áreas da pesquisa científica e da clínica (HERRERO et al, 2011).

Não foram encontradas pesquisas realizadas e publicadas no Brasil em torno da movimentação espontânea do lactente no que diz respeito à postura e aos ganhos motores de forma a compreender as diversidades culturais de nosso país, sendo este o objetivo futuro de desdobramento desta pesquisa.

O desenvolvimento neuromotor da criança, de acordo com Connolly, 2000; Hallal, Marques e Braccialli, 2008, é aspecto importante do desenvolvimento infantil. Segundo os autores as aquisições motoras, no primeiro ano de vida, são fator relevante no prognóstico do desenvolvimento global da criança, pois o período compreendido entre o nascimento e o final do primeiro ano de vida é considerado como um dos mais críticos no desenvolvimento infantil. Neste período, o desenvolvimento motor apresenta ritmo acelerado de mudanças que culminam nas funções de mobilidade, com a aquisição do engatinhar e da marcha independente, respectivamente aos 9 e 12 meses de idade. (CONNOLLY, 2000; HALLAL, MARQUES E BRACCIALLI, 2008).

O primeiro ano de vida, caracterizado por uma explosão de habilidades motoras e cognitivas está, intimamente, associado a um pré-natal adequadamente desenvolvido do qual exerce forte influencia sobre o desenvolvimento pós-natal estabelendo as bases biológicas para um desenvolvimento saudável. (ANDRACA et al, 1998)

Os autores dizem que as trocas de posturas, o desenvolvimento das reações de retificação e equilíbrio e a exploração do meio estão associados ao interesse do lactente em explorá-lo e de como será chamada sua atenção.

A evolução estático-motora do neonato até a adolescência depende da maturação do sistema nervoso central, sendo determinada por padrões, geneticamente, estabelecidos e estímulos ambientais. Esses estímulos apreendidos pelos órgãos dos sentidos são respondidos pelo cérebro como órgão de integração e coordenação, com reações complexas caracterizadas pelo desenvolvimento dos mecanismos reflexos da 
atitude ou postura e da manutenção desta, que permite ao homem erguer-se contra a gravidade e conservar seu equilíbrio que decorrem automaticamente. Essas dependem, em larga medida, da manipulação que a criança experimenta pela mãe - o mundo que está ao seu redor (FLEHMIG, 1987).

Assim uma estratégia eficaz e eficiente é a brincadeira, que deve ser adaptada de acordo com a faixa etária e as capacidades do lactente sem trabalhar apenas com o que ele já é capaz de fazer, e sim acrescentando novas posturas, regras, limites, intenção e concentração na atividade, (CONNOLLY, 2000; HALLAL, MARQUES E BRACCIALLI, 2008).

A concentração exigida da criança quando brinca, para Winnicott, (1975), mencionado por Rodrigues e Freitas, (2008), depende da confiança e do vínculo estabelecido com cuidador e o ambiente que se encontra:

[...] no brincar, a criança incorpora o movimento, relaciona-se com objetos e suas propriedades físicas. Desenvolve, progressivamente, as linguagens oral e gestual, conhece os conteúdos sociais (papéis, situações, valores, atitudes, limites definidos pelas regras) e sua relação com o universo social, e, nas mudanças de percepção culminantes de todas essas relações, conhece novas maneiras de experienciar o mundo.

(WINNICOTT,1975, apud, RODRIGUES E FREITAS, 2008)

Todo desenvolvimento motor realiza-se sempre sob uma ideal adaptação aos estímulos externos. Organismos e meio ambiente são dependentes um do outro. As fases motoras e os processos psíquicos e cognitivos influenciam-se reciprocamente de modo imediato manifestando-se, quase sempre, mediante modalidades comportamentais motoras, como por exemplo, pela mímica ou por meio de atitude corporal, daí terem função de comunicação com o meio ambiente. (FLEHMIG, 1987)

Abordamos em outro momento, deste estudo, questões apontadas por pesquisadores que são fundamentais na abordagem atual sobre o desenvolvimento infantil, e quem justificam a importância de um olhar significativo com relação aos aspectos externos que podem influenciar o desenvolvimento das crianças. Relembrando: dados maternos e paternos; moradia; dados da gestação; dados de parto; dados neonatais; condições de saúde e situação de estimulação. De acordo com dados da UNICEF, (2003), a vulnerabilidade da criança no período perinatal se dá por ocasião do parto e do pós-parto imediato.

Os diferentes problemas característicos do período neonatal são de grande importância devido as altas taxas de mortalidade que provocam e pelas sequelas que deixam. No Brasil a cada ano mais de 100 mil crianças não completam o seu primeiro ano de vida. Entre 2 mil e 3,5 mil mães morrem das consequências da falta de atendimento de qualidade durante a gravidez, o parto e o pós-parto. Os riscos para mãe e seu filho aumentam por falta de informação adequada sobre os cuidados necessários ou por falta de acesso a serviços de saneamento. (UNICEF, 2003). 
Dentre as principais causas de morbidade e mortalidade, também relacionadas ao lactente, estão a anóxia neonatal, o baixo peso ao nascer, o tétano neonatal e as infecções congênitas, segundo Rouquayrol (1994).

Segundo Sagre,(2002), todo recém-nascido que apresenta peso abaixo de 2.500 gramas deve ser considerado recém-nascido de baixo peso. Entretanto variações regionais importantes, bem como, como nível de renda e educação (instrução) materna influenciam o peso do bebê ao nascimento.

Outras causas de morbi-mortalidade podem ser citadas como as infecções congênitas, dentre elas a sífilis como mais frequente, assim como, morte causada por múltiplos patógenos, incluindo vírus, bactérias e fungos que causam desidratação. (ROUQUAYROL, 1994).

Infecções respiratórias agudas (IRA) também são comprometidas pela presença desses patógenos sendo que a grande maioria dessas infecções acometem crianças de menos de 2 anos de idade colaborando para uma das mais altas taxas no primeiro ano de vida (ROUQUAYROL, 1994).

A desnutrição energético-proteica (DEP) também afeta as crianças em suas formas primárias por falta absoluta de alimento e de forma secundária por outros faores externos como práticas inadequadas de alimentação e doenças infecciosas. (BRAZELTON, 1994).

O aleitamento materno oferece o alimento, nutricionalmente, mais adequado para o lactente e traz uma proteção importante contra a desnutrição, a diarreia, as IRA e a própria mortalidade infantil. (BRAZELTON, 1994).

Apesar desses fatores, as estatísticas apontadas pela Organização Mundial de Saúde (OMS, 2000) apontam para uma melhora no Brasil, sendo essas estatísticas mais favoráveis aos moradores da cidade a do interior. Os municípios de menor população, economicamente pouco desenvolvidos e distantes das capitais tendem a ter piores estatísticas. (OMS, 2000).

Medidas simples podem ser tomadas para incentivar o aleitamento materno, como, por exemplo, propiciar informação adequada às mães sobre seu benefício, preparar melhor os profissionais de saúde e equipe multiprofissional e desenvolver programas educacionais ampliando informações e levando conhecimento em torno da importância do aleitamento materno para as crianças. Porém, é possível que as crianças também tenham recebido uma alimentação (aleitamento) suficiente mas faltou-lhe a comunicação amorosa entre mãe e filho.

Segundo Brazelton, (1994), devido a falta de uma assistência carinhosa, o alimento ingerido pela criança passa pelo seu intestino sem ser digerido.

Quando uma mãe consegue fazer com que a criança ouse olhar para o seu rosto, ou aceite que este adulto cante para ela, embale-a ou aconchegue-a ao corpo no momento da alimentação, eles param de arquear o corpo ou de evitar o olhar das pessoas. A partir de então começam a ganhar peso e apresentar um 
desenvolvimento saudável. (BRAZELTON, 1994). A OMS e a UNICEF recomendam o mínimo de 4 meses de aleitamento materno. (OMS, 2000; UNICEF, 2001).

No que se refere à escolaridade da população brasileira, o Instituto Brasileiro de Geografia e Estatística (2001) aponta que as mulheres apresentam número médio de anos de escolaridade levemente superior ao dos homens. A diferença mais significativa emerge quando se comparam dados entre população branca e a parda e negra: 6,6 anos para brancos e 4,6 para pardos e negros na média para o país. (IBGE, 2001)

Dependendo do grau de escolaridade materna, do acesso às informações em saúde e do tempo dedicado aos cuidados da criança, esta tem um maior ou menos risco de adoecer. Mesmo vivendo em condições ambientais desfavoráveis as crianças que recebem melhores cuidados apresentam uma melhor condição de saúde. Noções de higiene pessoal, alimentação e de Âmbito doméstico contribuem na redução da contaminação da água, alimentos, mamadeiras e outros utensílios ligados às crianças (UNICEF, 2001).

\section{OBJETIVOS}

Com base nesses estudos o objetivo desta pesquisa foi avaliar o perfil dos lactentes moradores da comunidade de Paraisópolis atendidos em serviço de follow-up do Programa Einstein na Comunidade de Paraisópolis (PECP), em São Paulo, SP.

\section{MÉTODO}

Com relação à escolha do método de pesquisa e dos pacientes definiu-se por um estudo transversal, observacional e descritivo realizado em duas etapas: a) avaliação do lactente através da verificação do desempenho motor pela Escala Motora Infantil Alberta e b) aplicação de questionário feito aos cuidadores para investigação de informações perinatais, socioeconômicas, nutricionais e de ambientes de estimulação. Foram avaliados os lactentes com idade entre 4 e 6 meses, nascidos de parto único, com peso ao nascer de 2.500, a termo, idade gestacional entre 37 e 42 semanas, moradores da comunidade de Paraisópolis em serviço de follow-up do Programa Einstein na Comunidade de Paraisópolis (PECP), em São Paulo, SP.

- Critérios de inclusão e Exclusão:

Os critérios de inclusão foram: bebês entre 4 e 6 meses de vida, nascidos de parto único, com peso acima de 2.500 gramas ao nascer e com idade gestacional entre 37 e 42 semanas (SEGRE, 2000) moradores da comunidade de Paraisópolis e em serviço de follow-up do Programa Einstein na Comunidade de Paraisópolis (PECP).

Para os critérios de exclusão foram elencados: presença de doenças neurológicas, cardíacas ou malformações congênitas, detectadas no exame pediátrico ao nascer ou intercorrências neonatais importantes como distúrbios metabólicos, circular de cordão, anóxia, dentre outros. 
- Instrumento de Avaliação:

Foi utilizado como instrumento de avaliação a Escala Motora Infantil Alberta (ANEXO 1) devido a parâmetros considerados na introdução deste estudo.

O foco da observação é a avaliação da movimentação espontânea sequencial das crianças, do controle postural às posições supina, prona, sentada e ortostática. A administração do teste envolve manipulação mínima de manuseio.

A escala Alberta oferece a possibilidade de detectar, o mais cedo possível, qualquer desvio do desenvolvimento motor permitindo, assim, uma intervenção precoce para remediar ou minimizar os efeitos da disfunção.

A escala não traz nenhum perigo ou desconforto à criança (TECKLIN, 2002) e Segundo Piper e Darrah, (1998), o percentil 10, é sugerido como ponto de corte para classificar os lactentes quanto ao desempenho motor, ou seja, aqueles que apresentarem desempenho abaixo do percentil 10 são classificados como atrasos e aqueles acima, como não atrasos.

- Variáveis coletadas:

Com relação às variáveis coletadas foram listadas as variáveis que caracterizaram a população de estudo (APENDICE A)

- Análise de dados:

A análise de dados foi realizada através dos programas computacionais EXCEL onde elaborou-se um banco de dados com as informações coletadas em formulário, (APÊNDICE A)

- Considerações Éticas:

Os pesquisadores responsáveis assumiram que as informações seriam trabalhadas de forma coletiva não sendo possível a identificação dos sujeitos da pesquisa que poderiam a qualquer tempo se recusar a colaborar na coleta dos dados sem que haja prejuízo à sua permanência no serviço do programa. Os pais responsáveis pelos bebês assinaram Termo de Consentimento Livre e Esclarecido - TCLE - (APENDICE B).

\section{RESULTADOS E DISCUSSÃO}

Os Resultados apresentados, abaixo, procuram caracterizar os lactentes quanto ao desempenho neuromotor avaliado através da Escala Alberta e referente as variáveis biológicas e sociais. 
Tabela 1. Distribuição de lactentes segundo o sexo e a idade em meses

\begin{tabular}{lllllll}
\hline $\begin{array}{l}\text { Idade } \\
\text { em meses }\end{array}$ & Masculino & \multicolumn{2}{l}{ Feminino } & Total & \\
\hline & $\mathrm{N}$ & $\%$ & $\mathrm{~N}$ & $\%$ & $\mathrm{~N}$ & $\%$ \\
\hline $\mathbf{4}$ & 13 & 43,3 & 3 & 16,7 & 16 & 33,3 \\
$\mathbf{5}$ & 10 & 33,3 & 9 & 50,0 & 19 & 39,6 \\
$\mathbf{6}$ & 7 & 23,3 & 6 & 33,3 & 13 & 27,1 \\
TOTAL & 30 & 100,0 & 18 & 100,0 & 418 & 100,0 \\
\hline
\end{tabular}

Das 48 crianças que participaram deste estudo, o numero de meninos foi superior as de meninas sendo também, inversamente proporcional a sua idade. Em relação ao numero de meninas pode-se observar que a metade das meninas apresentava-se com idade de cinco meses. A média do grupo foi de 5,3 meses, a mediana de 5,4 e o desvio padrão de 0,8 .

Tabela 2. Número de consulta no pré-natal

\begin{tabular}{lll}
\hline $\begin{array}{l}\text { Número de consultas de } \\
\text { pré-natal }\end{array}$ & $\mathbf{N}$ & $\%$ \\
\hline$<6$ & 7 & 14,6 \\
\hline $\mathbf{6}$ a 10 & 33 & 68,7 \\
\hline 11 e mais & 7 & 14,6 \\
\hline Ignorado & 1 & 2,1 \\
TOTAL & 48 & 100,0 \\
\hline
\end{tabular}

É importante relatar que das 48 mães, todas realizaram consultas pré-natal (tabela não apresentada) e quanto aos numero de consultas indicado na tabela acima (tabela 2) 83,3\% das mães fizeram seis ou mais consultas (número suficiente indicado pelo Programa de Assistência Integral à Saúde da Mulher - PAISM e COSTA 2001) e apenas 14,6\% fizeram menos de seis consultas. Em apenas um caso não foi possível saber o numero de consulta, pois a entrevistada não era a mãe, não podendo responder a pergunta.

Tabela 3. Tipos de Intercorrência durante a gestação

\begin{tabular}{lll}
\hline $\begin{array}{l}\text { Tipo de intercorrência materna } \\
\text { Durante a gestação }\end{array}$ & $\mathbf{N}$ & $\mathbf{\%}$ \\
\hline Diabetes & 1 & 2,1 \\
Dor Abdominal & 1 & 2,1 \\
Hipertensão & 3 & 6,3 \\
Infecção do trato Urinário & 5 & 10,4 \\
Sangramento & 1 & 2,1 \\
Sem intercorrências & 37 & 77,1 \\
TOTAL & 48 & 100,0 \\
\hline
\end{tabular}

Pode-se perceber com os dados expostos que em aproximadamente $80 \%$ dos casos, não ocorreram intercorrências e dos $20 \%$ restante, apenas $10 \%$ relatam infecção do trato urinário como intercorrência, demonstrando que não houve necessidade de internação hospitalar. 
Tabela 4. Distribuição dos lactentes segundo idade gestacional

\begin{tabular}{lll}
\hline $\begin{array}{l}\text { Idade Gestacional } \\
\text { (em semanas) }\end{array}$ & $\mathbf{N}$ & $\mathbf{\%}$ \\
\hline $\mathbf{3 7}$ & 3 & 6,3 \\
$\mathbf{3 8}$ & 4 & 8,3 \\
$\mathbf{3 9}$ & 18 & 37,5 \\
$\mathbf{4 0}$ & 19 & 39,6 \\
$\mathbf{4 1}$ & 3 & 6,3 \\
$\mathbf{4 2}$ & 1 & 2,1 \\
TOTAL & 48 & 100,0 \\
\hline
\end{tabular}

A maioria das crianças, cerca de $80 \%$ nasceu entre 39 e 40 semanas, tendo sido classificadas como termo. Nos extremos da idade gestacional, ainda dentro dos critérios de inclusão do estudo, observaram-se poucos lactentes.

Tabela 5. Distribuição dos lactentes segundo peso ao nascimento (PN)

\begin{tabular}{lll}
\hline $\begin{array}{l}\text { Peso ao nascimento } \\
\text { (em gramas) }\end{array}$ & $\mathbf{N}$ & \% \\
\hline $\mathbf{2 . 5 0 0}-\mathbf{3 . 0 0 0}$ & 20 & 41,7 \\
$\mathbf{3 . 0 0 0}-\mathbf{3 . 5 0 0}$ & 15 & 31,3 \\
$\mathbf{3 . 5 0 0}-\mathbf{4 0 0 0}$ & 12 & 25,0 \\
Acima de 4.000 & 1 & 2,1 \\
TOTAL & 48 & 100,0 \\
\hline
\end{tabular}

A média de peso foi de 3.167,2 gramas e o desvio padrão foi de 414,6. A maior parte do grupo, $73 \%$, encontra-se com peso entre 2.500 e 3.500 gramas. Ainda assim, 41,7\% dos lactentes apresentaram PN insuficiente (NOBREGA, 1989).

Tabela 6. Diagnóstico Neonatal

\begin{tabular}{lll}
\hline $\begin{array}{l}\text { Diagnóstico de Peso ao nascer } \\
\text { (Por idade gestacional) }\end{array}$ & $\mathbf{N}$ & $\boldsymbol{\%}$ \\
\hline AIG & 39 & 81,2 \\
GIG & 1 & 2,1 \\
\hline PIG & 8 & 16,7 \\
TOTAL & 48 & 100,0 \\
\hline
\end{tabular}

Na tabela acima, (tabela 6), observa-se que os lactentes avaliados foram recém-nascidos adequados para a idade gestacional (AIG). Entretanto 8 deles apresentaram PN em relação à idade gestacional, abaixo do esperado.

Tabela 7. Intercorrência no Parto

\begin{tabular}{lll}
\hline Tipo de Intercorrência & N & \% \\
\hline Apresentação pélvica & 1 & 2,1 \\
\hline Pouca dilatação do canal de parto & 1 & 2,1 \\
Sem dilatação do canal de parto & 6 & 12,5 \\
Sofrimento fetal & 2 & 4,2 \\
Sem intercorrência & 37 & 77,1
\end{tabular}




\begin{tabular}{lll} 
Ignorado & 1 & 2,1 \\
\hline TOTAL & 48 & 100,0 \\
\hline
\end{tabular}

Na tabela acima (tabela 7) pode-se observar que quase $80 \%$ dos bebês não apresentam nenhuma intercorrência ao nascer. Entretanto $14,6 \%$ das mães apresentaram pouca ou nenhuma dilatação no momento do parto. Deve-se destacar que dois bebês apresentaram sofrimento fetal e o outro não estava na posição correta para nascer.

Tabela 8. Intercorrência perinatais

\begin{tabular}{lll}
\hline Tipo de Intercorrência & N & \% \\
\hline Celulite torácica & 1 & 2,1 \\
Crise convulsiva & 1 & 2,1 \\
Síndrome de Aspiração Meconial & 5 & 10,4 \\
Sem intercorrência & 41 & 85,4 \\
TOTAL & 48 & 100,0 \\
\hline
\end{tabular}

Na tabela acima, (tabela 8), observa-se que mais de $85 \%$ dos bebês não apresentaram nenhuma intercorrência perinatal. No entanto 14,6\% deles apresentaram intercorrências importantes, sendo na sua maioria (5 casos) a Síndrome de Aspiração Meconial. É importante salientar que nenhum lactente que participo deste estudo apresentou alteração tônica importante que levasse a criança a ser encaminhada para uma avaliação neurológica.

Tabela 9. Cuidador principal

\begin{tabular}{lll}
\hline Cuidador Principal & N & \% \\
\hline Avó & 2 & 4,2 \\
Mãe & 43 & 89,6 \\
Tia & 3 & 6,2 \\
TOTAL & 48 & 100,0 \\
\hline
\end{tabular}

Aproximadamente $90 \%$ dos cuidadores principais são as mães. Neste caso entendeu-se como cuidador principal aquele que passa o maior período com a criança podendo atender as suas necessidades.

Tabela 10. Idade Materna

\begin{tabular}{lll}
\hline Faixas de Idade Materna & $\mathbf{N}$ & \% \\
\hline$<\mathbf{1 5}$ & - & - \\
$\mathbf{1 5}$ a 18 & 11 & 22,9 \\
$\mathbf{1 9}$ a 21 & 11 & 22,9 \\
$\mathbf{2 2}$ a 25 & 10 & 20,8 \\
$\mathbf{2 6}$ a 30 & 8 & 16,7 \\
$\mathbf{3 0}$ a 34 & 5 & 10,4 \\
35 e mais & 3 & 6,3 \\
TOTAL & 48 & 100,0 \\
\hline
\end{tabular}


A maioria das mães apresenta-se com intervalo entre 19 e 30 anos de idade, obtendo uma média de idade igual a 23,7. A média apresenta-se fora da faixa de risco para engravidar, podendo ser comparada a outros estudos relacionados ao tema. No entanto é uma média de idade baixa, quando se considera que estas mães poderiam estar ainda estudando e iniciando-se no mercado de trabalho.

Tabela 11. Escolaridade Materna

\begin{tabular}{llc}
\hline $\begin{array}{l}\text { Escolaridade Materna } \\
\text { (em anos de estudo) }\end{array}$ & $\mathbf{N}$ & \% \\
\hline$<\mathbf{4}$ & 7 & 14,6 \\
$\mathbf{4}$ a 8 & 26 & 54,2 \\
9 a 11 & 14 & 29,2 \\
12 e mais & - & - \\
Ignorado & 1 & 2,1 \\
TOTAL & 48 & 100,0 \\
\hline
\end{tabular}

O nível de escolaridade materna observado na tabela acima (tabela11) foi baixo, pois 14,6\% das mães podem ser consideradas analfabetas funcionais e ainda certa de $70 \%$ delas não atingem o nível médio de escolaridade.

Tabela 12. Doença da criança, relatada pela mãe, no dia da avaliação

\begin{tabular}{llc}
\hline Doenças na época da avaliação & N & \% \\
\hline Anemia Falciforme & 2 & 4,2 \\
Bronquiolite & 1 & 2,1 \\
Conjuntivite & 1 & 2,1 \\
Gripe & 2 & 4,2 \\
Otite Média Aguda & 1 & 2,1 \\
Refluxo Gastro-Esofágico (RGE) & 1 & 2,1 \\
Sem doença & 40 & 83,4 \\
TOTAL & 48 & 100,0 \\
\hline
\end{tabular}

Os lactentes deste estudo apresentaram poucas doenças na época da avaliação, porém em nenhum caso houve impossibilidade de avaliar o bebê por conta do mesmo apresentar algum estado de humor inadequado para a avaliação. Daqueles que apresentaram alguma doença, tem-se que 8,4 tiveram doenças alérgicas ou respiratórias.

Tabela 13. Número de crianças relacionadas à faixa de aleitamento materno exclusivo apresentado em dias.

\begin{tabular}{llc}
\hline $\begin{array}{l}\text { Faixas de aleitamento } \\
\text { Materno exclusivo } \\
\text { (apresentação em dias) }\end{array}$ & $\mathbf{N}$ & $\%$ \\
\hline Não foi amamentado & & \\
\hline Até 30 & 1 & 2,1 \\
31 a 60 & 3 & 6,2 \\
\hline 61 a 90 & 4 & 8,3 \\
91 a 120 & 4 & 8,3 \\
\hline $\mathbf{1 2 0}$ e mais & 13 & 27,1 \\
\hline & 23 & 47,9 \\
\hline
\end{tabular}


Metade da amostra dos bebês recebeu aleitamento materno exclusivo por mais de 120 dias, ou seja, os quatro meses recomendados como tempo mínimo pela Organização Mundial de Saúde, (2000) e a UNICEF, (2001). A média brasileira segundo o Ministério da Saúde (1999) é de 33,7 dias.

Tabela 14. Local de permanência do bebê

\begin{tabular}{lll}
\hline Local & N & \% \\
\hline Banheira & 2 & 4,2 \\
Berço & 6 & 12,5 \\
Cama & 18 & 37,5 \\
Carrinho & 8 & 16,7 \\
Chão & 8 & 16,7 \\
Colchonete & 1 & 2,1 \\
Colo & 2 & 4,2 \\
Sofá & 2 & 4,2 \\
Ignorado & 1 & 2,1 \\
TOTAL & 48 & 100 \\
\hline
\end{tabular}

Com relação ao local predominante de permanência do bebê durante os períodos em que está desperto, pode-se notar que quase $40 \%$ deles permanecem na cama dos pais e $43,8 \%$ permanece em locais que não possibilitam movimento (berço, banheira, carrinho, colo e sofá).

Tabela 15. Distribuição dos lactentes segundo percentil obtido na Escala Alberta

\begin{tabular}{lll}
\hline Percentil & N & \% \\
\hline$<$ P10 & 7 & 14,6 \\
P10 a P25 & 18 & 37,5 \\
P25 a P50 & 13 & 27,1 \\
P50 a P90 & 7 & 14,6 \\
>P90 & 3 & 6,3 \\
TOTAL & 48 & 100,0 \\
\hline
\end{tabular}

Levando em consideração que bebês localizados abaixo do percentil 10 apresentam uma chance maior de restrição ao desenvolvimento neuromotor, na Tabela acima, (tabela 15), 7 (sete) lactentes (14,6\%) apresentaram restrição. Além disso, se estes lactentes são incluídos, certa de $80 \%$ deles estão abaixo da média esperada para a idade.

\section{DISCUSSÃO}

A discussão dos achados aponta para novidades bem como aprofundamento na pesquisa sobre a temática.

Nas pesquisas realizadas os autores entendem por desenvolvimento motor as mudanças ocorridas nos padrões de movimento que estão presentes ao longo da vida, onde observamos o aprimoramento das 
ações, a evolução de atividades rotineiras, fundamentais e específicas (CONNOLLY, 2000; HALLAL, MARQUES E BRACCIALLI, 2008). Muito desta evolução está em cada momento da vida de recémnascidos e lactentes, que além de experimentarem o movimento, divertem-se e passam o tempo entretidos nas vivências inovadoras e prazerosas (Trindade, 2007).

Gesell,(1945), já referenciava questões biológicas e ambientais como determinantes do desenvolvimento, além de acrescentar que o contexto oferece oportunidades de organização e desenvolvimento às crianças.

Para que se possa realizar uma discussão à luz dos resultados encontrados preferiu-se por sequenciar na mesma ordem em que listamos os resultados já apresentados. Assim:

\section{a) Com relação à distribuição de lactentes segundo o sexo e a idade em meses}

O numero de meninos foi superior as de meninas sendo também, inversamente proporcional a sua idade.

\section{b) Com relação ao número de consulta no pré-natal}

Todas as mães realizaram consulta pré-natal sendo que $83,3 \%$ das mães fizeram seis ou mais consultas (número suficiente indicado pelo Programa de Assistência Integral à Saúde da Mulher - PAISM e COSTA 2001) e apenas 14,6\% fizeram menos de seis consultas. Embora a qualidade do tratamento não tenha sido observado.

\section{c) Ao que se refere aos tipos de Intercorrência durante a gestação}

Em, aproximadamente, $80 \%$ dos casos, não ocorreram intercorrências e dos $20 \%$ restante, apenas $10 \%$ relatam infecção do trato urinário como intercorrência, demonstrando que não houve necessidade de internação hospitalar.

\section{d) Sobre a distribuição dos lactentes segundo idade gestacional}

Cerca de $80 \%$ nasceu entre 39 e 40 semanas, tendo sido classificadas como termo.

\section{e) Com relação à distribuição dos lactentes segundo peso ao nascimento (PN)}

A média de peso foi de 3.167,2 gramas. A maior parte do grupo, 73\%, encontra-se com peso entre 2.500 e 3.500 gramas. Ainda assim, 41,7\% dos lactentes apresentaram PN insuficiente.

\section{f) Sobre o diagnóstico Neonatal}

Os lactentes avaliados foram recém-nascidos adequados para a idade gestacional (AIG). Entretanto, 8 deles apresentaram PN em relação à idade gestacional, abaixo do esperado.

\section{g) Com relação às Intercorrência no Parto}

Quase $80 \%$ dos bebês não apresentam nenhuma intercorrência ao nascer. Entretanto, 14,6\% das mães apresentaram pouca ou nenhuma dilatação no momento do parto. Dois bebês apresentaram sofrimento fetal e o outro não estava na posição correta para nascer.

h) Nas intercorrência perinatais 
Mais de $85 \%$ dos bebês não apresentaram nenhuma intercorrência perinatal. No entanto 14,6\% deles apresentaram intercorrências importantes, sendo na sua maioria (5 casos) a Síndrome de Aspiração Meconial. Esta síndrome está relacionada com o sofrimento fetal o que poderia sugerir uma assistência não adequada oferecida às mães durante o período da gestação e no momento do parto. É importante salientar que dos lactentes que apresentaram história de SAM, (5), apenas 1 caso foi encontrado a restrição do desenvolvimento neuromotor;

\section{i) Sobre o Cuidador principal e a relação da idade materna}

Aproximadamente $90 \%$ dos cuidadores principais eram as mães e em sua maioria apresenta-se com intervalo de idade entre 19 e 30 anos obtendo uma média de idade igual a 23,7.

\section{j) No que diz respeito à escolaridade Materna}

Mais que a metade das 48 mães tinha apenas de 4 a 8 anos de estudo sendo esta classificação considerada como Ensino Fundamental (De $1^{\mathrm{a}}$ a $9^{\circ}$ ano) segundo o Ministério da Educação e Cultura MEC (2007).

k) Sobre a doença da criança, relatada pela mãe, no dia da avaliação.

Os lactentes apresentaram poucas doenças na época da avaliação, porém em nenhum caso houve impossibilidade de avaliar o bebê por conta do mesmo apresentar algum estado de humor inadequado para a avaliação.

1) Quando pesquisado sobre o número de crianças relacionadas à faixa de aleitamento materno exclusivo apresentado em dias.

Metade da amostra dos bebês recebeu aleitamento materno exclusivo por mais de 120 dias, ou seja, os quatro meses recomendados como tempo mínimo pela Organização Mundial de Saúde, (2000) e a UNICEF, (2001). A média brasileira segundo o Ministério da Saúde (1999) é de 33,7 dias. O desenvolvimento infantil depende muito do vínculo materno estabelecido desde o momento da gravidez (GARSD, 2003; BRAZELTON, 1994; FLEHMIG, 1987). O aleitamento materno exclusivo intensifica o vínculo estabelecido e o reforça. Neste estudo encontrou-se um tempo médio de amamentação maior comparado com a média de dias da população brasileira o que é preciso considerar que a maior parte das mães eram as cuidadores principais dos bebês.

\section{m) Sobre o local de permanência do bebê}

Durante os períodos em que está desperto quase $40 \%$ deles permanecem na cama dos pais e 43,8\% permanece em locais que não possibilitam movimento (berço, banheira, carrinho, colo e sofá). Esses dados nos mostram que a maior parte dos bebês permanece em locais de pouca estimulação para sua movimentação ou que até mesmo trazem risco para o próprio como a cama dos pais e o sofá. É preciso lembrar que a amostra deste estudo se constituiu de bebês de 4 a 6 meses. De acordo com Flehmig, (1994), 
os bebês nesta faixa etária não estariam arrastando ou engatinhando. Entretanto aqueles que poderiam adquirir estes comportamentos encontrariam limitações oferecidas pelo ambiente.

\section{n) E sobre a distribuição dos lactentes segundo percentil obtido na Escala Alberta}

7 (sete) lactentes $(14,6 \%)$ apresentaram restrição (levando em consideração que bebês localizados abaixo do percentil 10 apresentam uma chance maior de restrição ao desenvolvimento neuromotor de acordo com a Escala Alberta).

\section{CONSIDERAÇÕES FINAIS}

O objetivo desta pesquisa foi avaliar o perfil dos lactentes moradores da comunidade de Paraisópolis atendidos em serviço de follow-up do Programa Einstein na Comunidade de Paraisópolis (PECP), em São Paulo. Com base nos resultados da pesquisa permitiu-se concluir que a maioria dos lactentes avaliados apresentou abaixo da média no que se refere ao desempenho motor nos permitindo, a partir desse dado, considerar que a maioria desses bebês permanece em locais que não possibilitam movimento (berço, banheira, carrinho, colo e sofá) concordando com a literatura que crianças que crescem em ambientes carentes de estímulos não apresentam facilidade para o aprendizado motor uma vez que não possuem estruturas operacionais aptas a equacionarem novos eventos e sequências. 


\section{REFERÊNCIAS}

AMORIM RCA, LAURENTINO GEC, BARROS KMFT, FERREIRA ALPR, FILHO AGM, RAPOSO MCF. Programa de saúde da família: proposta para identificação de fatores de risco para o desenvolvimento neuropsicomotor. Rev Bras Fisioterapia. 2010; 13(6): 506-13.

ANDRACA, I; PINO, P; LA PARRA, A; RIVIEIRA Y Marcela, F. Factores de riesgo para el desarollo psicomotor em lactentes nacidos em optimas condiciones biológicas. Em Pauta - Revista de Saúde Pública, São Paulo, 32(2) 128-147. Abril 1998

BRAZELTON, T.B. Momentos decisivos do desenvolvimento infantil. São Paulo: Martins Fontes, v1, p.347-352, 1994.

CAPUTE AJ. Normal gross motor development: the influences of races, sex and socio- economic status. Dev Med Child Neurol 1985; 27: 635-43.

CONNOLLY K. Desenvolvimento motor: passado, presente e futuro.Rev Paul Educ Fis. 2000; 3: 6-15. DE VRIES MW. Babies brain and culture: optimizing neurodevelopment on the savanna. Acta Pediatric Suppl. 1999; 429:43-8.

FLEHMIG, I. Desenvolvimento normal e seus desvios no lactente. Rio de Janeiro, São Paulo: Livraria Atheneu, v1, p.09-45, 1987.

GANDRA YR. O pré-escolar de dois a seis anos de idade e o seu atendimento. Rev. Saúde Pública, v.15, supl.0, São Paulo, dez. 1981.

GESELL, A. The embriology of behavior. New York, Harper e Brothers, 1945

HAIDAR FH, OLIVEIRA UF, NASCIMENTO LFC. Escolaridade materna: correlação com os indicadores obstétricos. Cad. Saúde Pública, Rio de Janeiro, 17(4):1025-1029, jul-ago, 2001.

HALLAL CZ, MARQUES NR, BRACCIALLI LMP. Aquisição de habilidades funcionais na área de mobilidade em crianças atendidas em um programa de estimulação precoce. Rev. bras. crescimento desenvolv. hum., 18(1): 27-34, 2008.

HERRERO, D. et al. Escala de Desenvolvimento Motor em Lactentes. Rev. bras. crescimento desenvolv. hum., 21(1): 121-132, 2011.

IBGE. Net, SP, 2001. Mortalidade infantil. Disponível em <www.saude.sp.gov.br> . Acesso em 15.maio.2015.

PIPER MC, DARRAH J. Alberta Infant Motor Scale. Philadelphia: WB Sauders; 1994.

REZENDE MA, BETELI VC, SANTOS JLF. Follow-up of the child's motor abilities in day-care centers and pre-schools. Rev. Latino-Am. Enfermagem . 2010; 13(5): 619-625.

RODRIGUES C, Freitas, D. Educação física e educação infantil: uma reflexão teórica. Diálogos possíveis. Janeiro/ junho, 2008. Disponível em: <www.fsba.edu.br/dialogospossiveis>. Acesso em $8 \mathrm{dez}$ 2011. 
ROUQUAYROL, M.Z Epidemiologia e Saúde. Rio de Janeiro: MEDSI, 527 p. 1994.

SANTOS DCC. Desenvolvimento motor durante o primeiro ano de vida: comparação entre lactentes americanos e brasileiros. Tese de doutorado. Campinas. 2001

SEGRE, C.A.M. Perinatologia - Fundamentos e prática. São Paulo: Savier; 2002.

SILVA PL, Santos DCC, Gonçalves VMG. Influência de práticas maternas no desenvolvimento motor de lactentes do $6^{\circ}$ ao $12^{\circ}$ meses de vida. Rev. bras. fisioter. 2006, 10(2): 225-231.

UNICEF, net, SP. 2001.2003 Desmame adequado. Disponível em<www.unicef.br> . Acesso em 06.mar.2015.

WALLON. H. A evolução psicológica da criança. Lisboa, Edições 70, 1998.

WINNICOTT, D.W. O brincar e a realidade. Rio de Janeiro: Imago, 1975 
ANEXOS

Anexo 1 - Escala Motora Infantil Alberta

\section{ESCALA MOTORA INFANTIL ALBERTA}

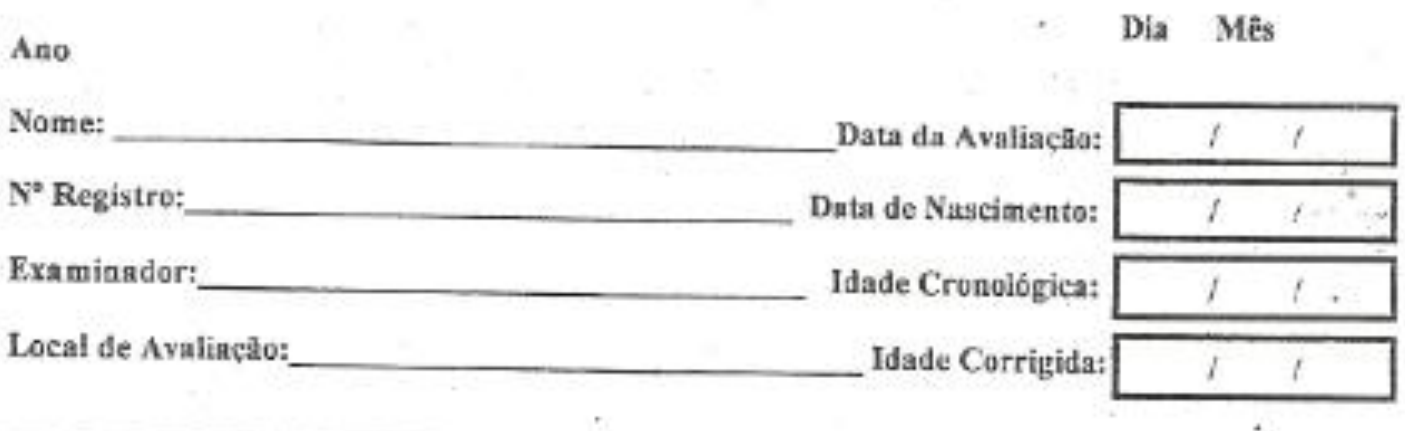

\begin{tabular}{|l|l|l|l|}
\hline & $\begin{array}{l}\text { Itens Anteriores } \\
\text { Creditados }\end{array}$ & $\begin{array}{l}\text { Itens Creditados } \\
\text { aa Janela }\end{array}$ & Escure da Subescala \\
\hline Prono & & & \\
\hline Sapino & & & \\
\hline Sentado & & & \\
\hline Em pé & & & \\
\hline
\end{tabular}

Escore Total:

Percentil:

Comentários e Recomendaçoes: 
ESCALA ALBERTA

9

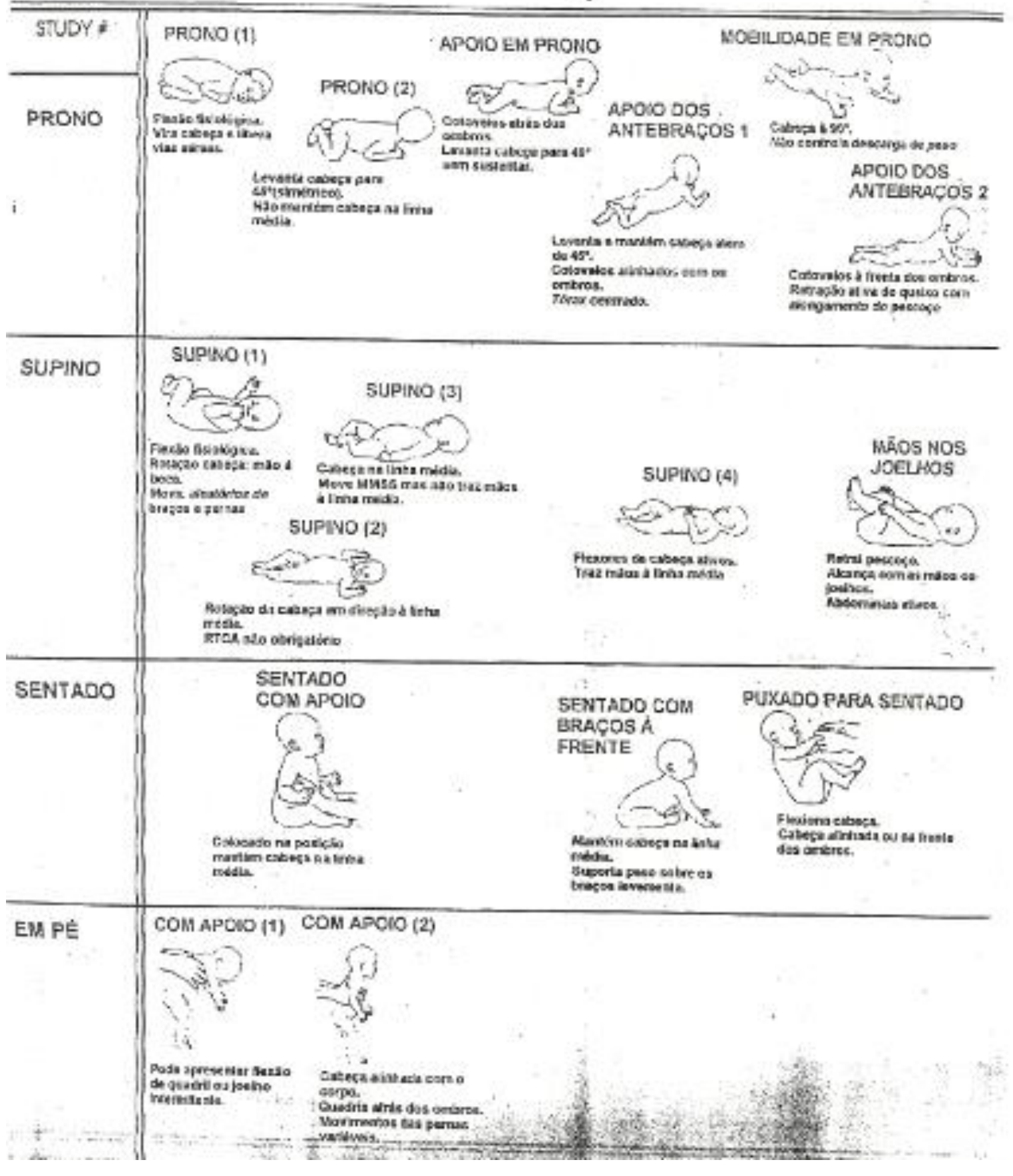




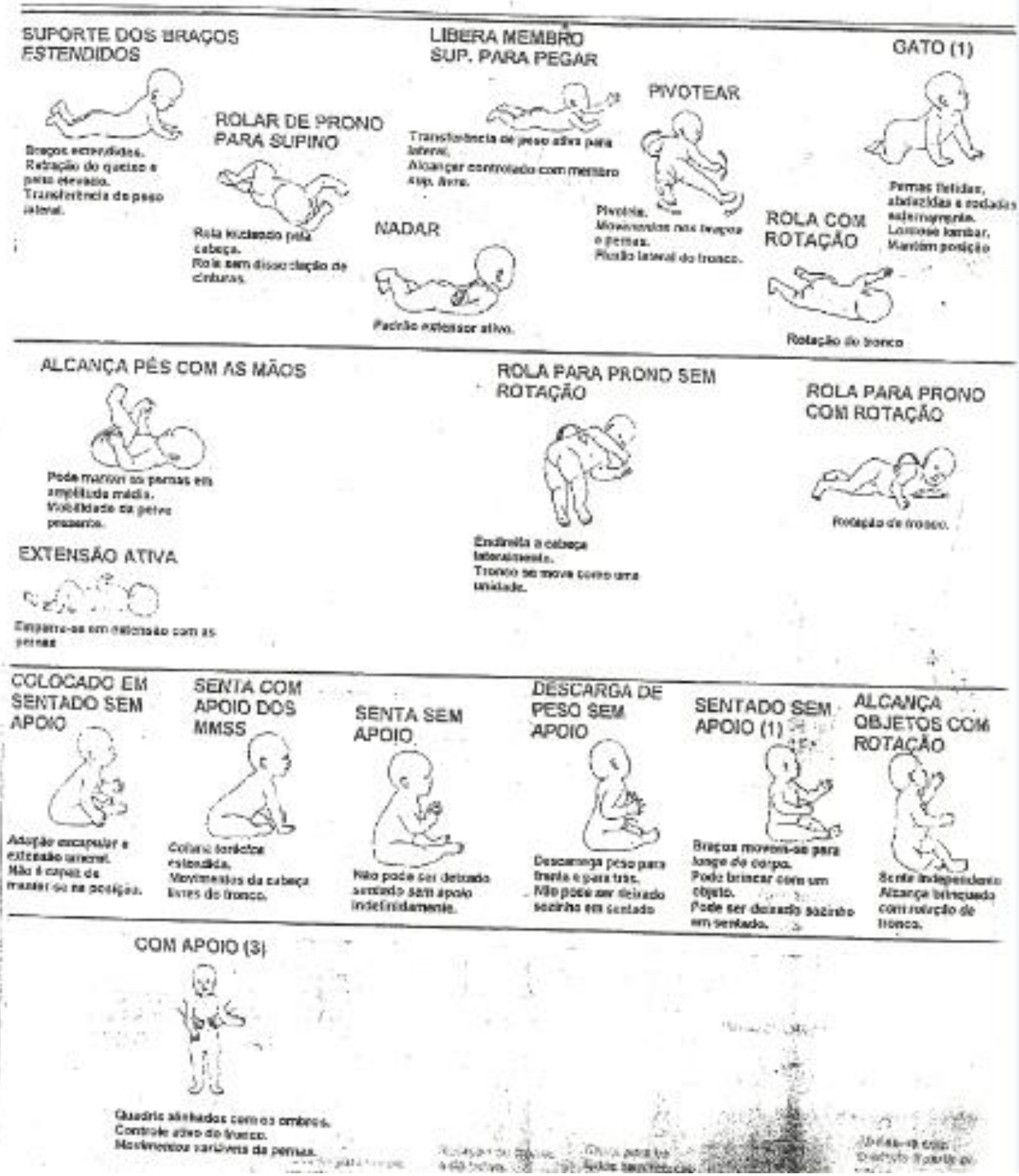



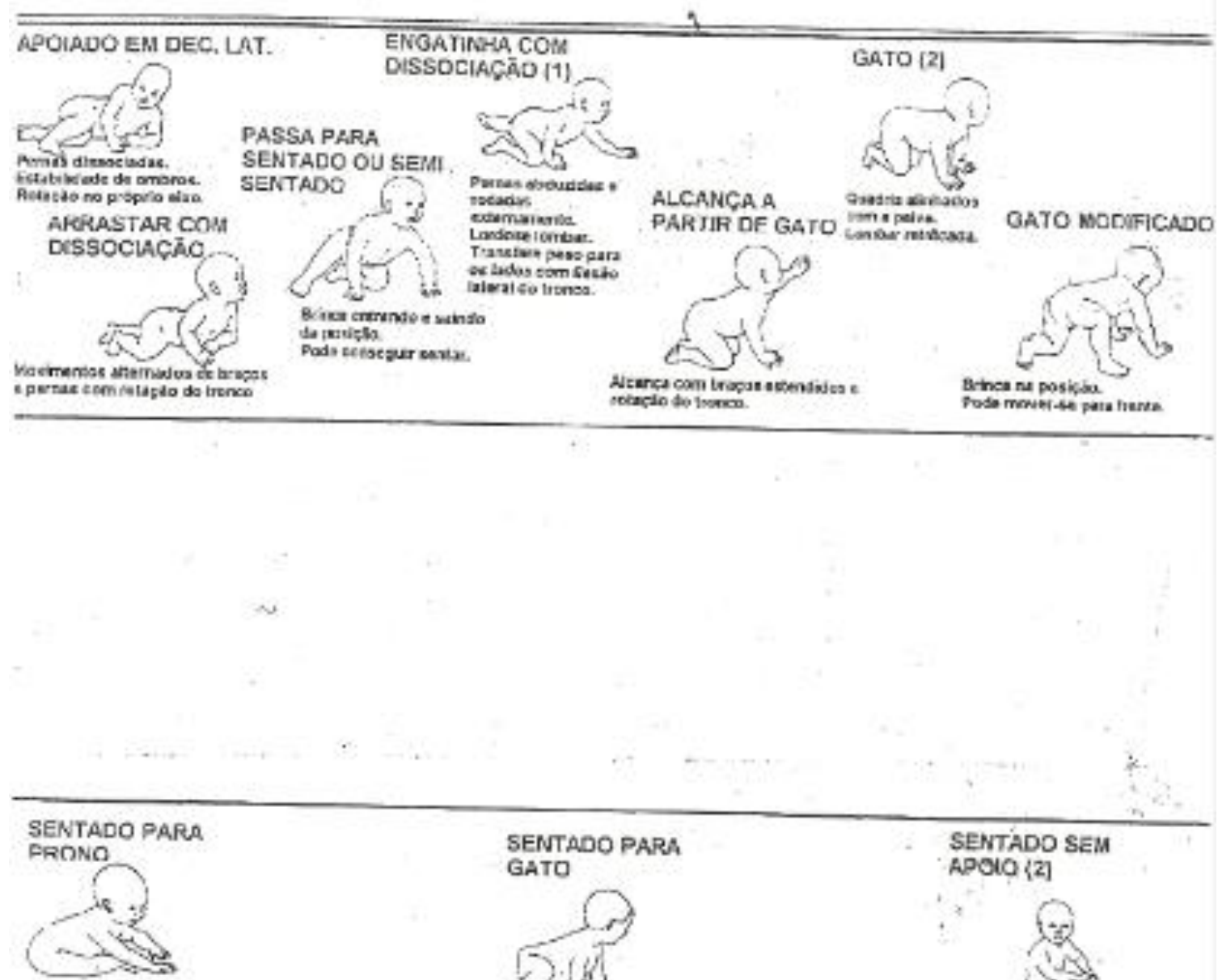

carace Musa.

Preasinatioas.

\section{s.}

(20)

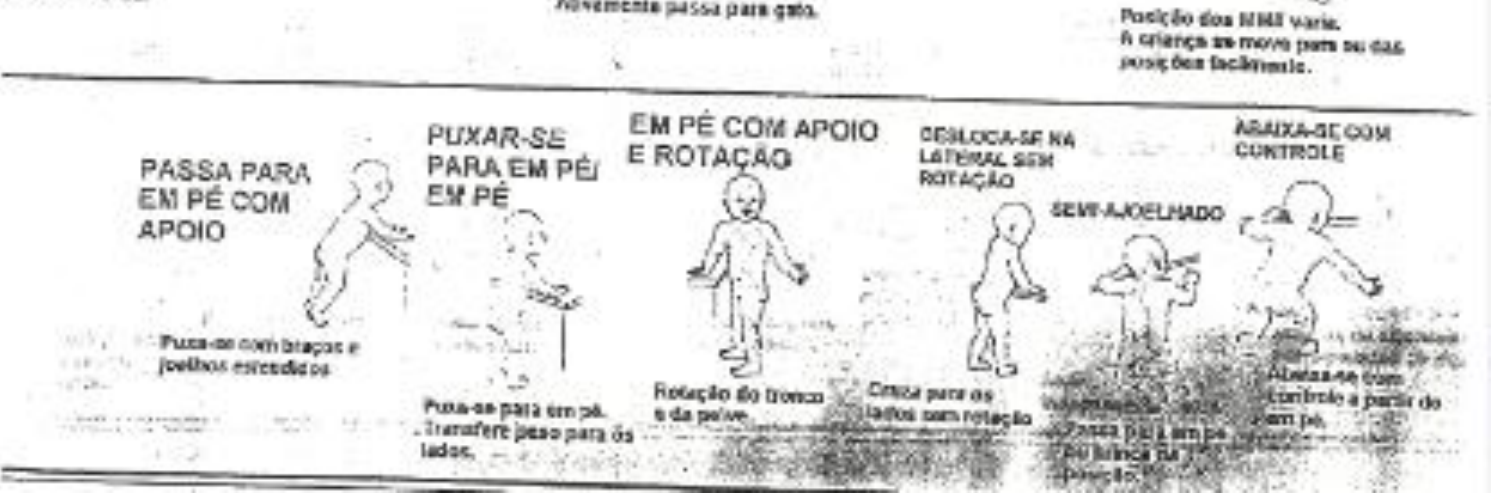


ENGATINHAR COM

DISSOCIAÇĀO

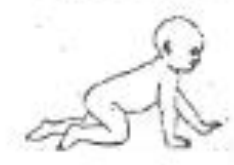

Coluns larnber retificedic.

Engaeinha com rocagso de

troneo.

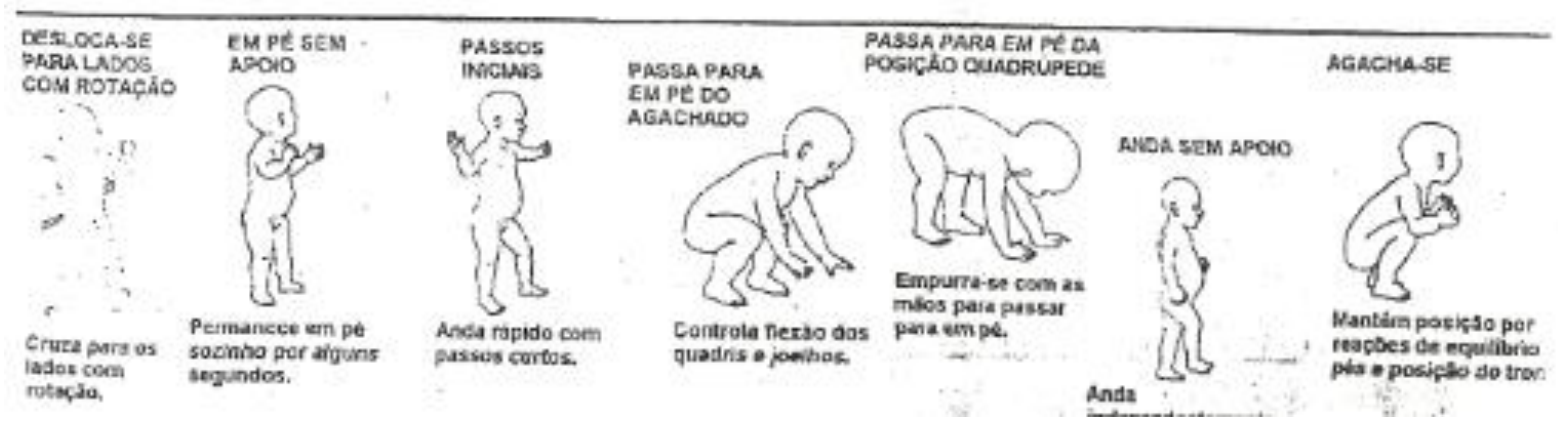

